\title{
Assistive devices: usage in patients with rheumatoid arthritis
}

\author{
I. G. de Boer • A. J. Peeters • H. K. Ronday • \\ B. J. A. Mertens • T. W. J. Huizinga • \\ T. P. M. Vliet Vlieland
}

Received: 21 April 2008 /Revised: 3 July 2008 /Accepted: 23 July 2008 / Published online: 26 August 2008

(C) The Author(s) 2008. This article is published with open access at Springerlink.com

\begin{abstract}
We describe the usage of various assistive devices and identify factors associated with usage in patients with rheumatoid arthritis (RA). A cross-sectional, multicentre study was performed in three outpatient rheumatology clinics in the Netherlands. Two hundred forty patients with RA participated in the study. The main measures were questionnaires and a semi-structured interview regarding the possession and usage of 21 common assistive devices in the ISO9999 categories orthopaedic footwear, personal care, mobility, household and adaptations for housing. Potential factors associated with usage included sociodemographic variables, health status, quality of life, coping strategies, selfefficacy, outcome expectations and satisfaction. Out of 240 patients, $213(89 \%)$ had one or more assistive devices in possession (median number of devices 3.0, interquartile range 3.0). The proportions of patients never using a device in possession varied between $8 \%$ for orthopaedic insoles and $23 \%$ for grab bars. The main factors related to usage varied among categories, but common determinants were a specific
\end{abstract}

I. G. de Boer · T. W. J. Huizinga • T. P. M. Vliet Vlieland $(\bowtie)$

Department of Rheumatology (C1-R),

Leiden University Medical Center,

P.O. Box 9600, 2300 Leiden, The Netherlands

e-mail: t.p.m.vliet_vlieland@lumc.nl

\section{A. J. Peeters}

Department of Rheumatology, Reinier de Graaf Gasthuis,

Delft, The Netherlands

H. K. Ronday

Department of Rheumatology,

Haga-Leyenburg Teaching Hospital,

The Hague, The Netherlands

B. J. A. Mertens

Department of Medical Statistics, LUMC,

Leiden, The Netherlands impairment or disability, satisfaction with the device or related services, self-efficacy and the number of devices in possession. In conclusion, in patients with RA, possession rates are high, with $23 \%$ or less of the devices in possession being abandoned. Overall, satisfaction rates were high. Factors associated with usage varied among categories and comprised, apart from the number of devices in possession and variables related to health status, also aspects of satisfaction with the device or related services or self-efficacy. The latter findings underline the need for a systematic evaluation of the outcomes of assistive devices by prescribing health professionals or suppliers in every individual case.

Keywords Assistive devices - Compliance .

Cross-sectional study · Disability $\cdot$ Rheumatoid arthritis

\section{Introduction}

Assistive devices are frequently prescribed in patients with rheumatoid arthritis (RA), with the aim of improving or maintaining the patient's functional ability and independence. $[1,2]$ A pre-requisite for assistive devices to be effective is, apart from their possession, their actual usage. Until now, both the possession of assistive devices and the adherence with their use are poorly studied.

Concerning possession, the proportions of RA patients having one or more assistive devices varies between $34 \%$ and $78 \%$ in the literature [3-7]. Variable possession rates may be due to differences in patient selection, the number and nature of the assistive devices being studied and the country or health care system where the study was conducted.

In studies focusing on the usage of assistive devices, the overall usage rate for assistive devices varied between $40 \%$ and $91 \%[2,3,7-10]$. It was found that the usage of 
assistive devices was associated with higher age $[2,7,11]$, more severe disease $[2,3,7,12]$, more disability $[2,3,7,12]$ and a beneficial effect or positive evaluation of the device.[2, $7,9,12]$ In these studies, a number of factors which are likely to be relevant for the actual usage of adaptive devices, such as the process of prescription and provision and the patient's evaluation of the design and comfort of adaptive devices, has not been taken into account.

The aim of the present study was to describe the usage of assistive devices and to identify factors contributing to usage in an unselected population of patients with RA. More insight into the actual usage of various assistive devices in daily practice, as well as factors related to their usage and non-usage could help rheumatologists and health professionals to define clear indications for their prescription or the provision of alternative solutions to relieve symptoms or reduce disability.

\section{Patients and methods}

\section{Patients}

This study was a multicentre, cross-sectional study performed between 2002 and 2004 in the rheumatology outpatient clinics of three hospitals (Leiden University Medical Center, LUMC; Haga-Leyenburg Teaching Hospital, The Hague; and Reinier de Graaf Hospital, Delft). In the Netherlands, the prescription process of assistive devices in patients with RA usually concerns the rheumatologist, the clinical nurse specialist, the occupational therapist or, less frequently, the physical therapist. In all three centres, patients had access to a clinical nurse specialist. In addition, patients could be referred to an occupational therapist or physical therapist in primary care or in a rehabilitation centre or to a multidisciplinary team care programme of the Rheumatology Clinic of the LUMC if considered necessary by their treating rheumatologist.

Inclusion criteria were (1) a diagnosis of RA according to the 1987 American College of Rheumatology criteria [13] and (2) being fluent in Dutch. There were no exclusion criteria. In the three hospitals, registries of patients with a clinical diagnosis of RA were sorted in ascending order by the day of the month of the patients' birthdays or by the date of their forthcoming visit to the outpatient clinic. The registries comprised $765,1,122$ and 360 patients in the three hospitals, respectively. Recruitment was done until the target number of 250 was reached. First, the diagnosis of RA was verified in the medical records by the principal investigator (IGB). Second, eligible patients were contacted by telephone to check whether they were able to communicate in Dutch and subsequently invited to participate in the study. Of the eligible patients who could not be reached by phone or declined participation, age, gender and disease duration were recorded. All participants were required to sign the informed consent. The protocol received ethical approval from the Medical Ethics Committees of all three participating hospitals.

\section{Measurements}

Data on the presence of an impairment or activity limitation related to the specific assistive device and the possession and usage of that device were gathered by means of a semistructured interview, which was carried out in the outpatient clinics of the participating hospitals. During this visit, a 28joint count [14] was performed. Both the semi-structured interview and the joint count were done by a trained assessor, the principal investigator of the study (IGB).

In addition, participants filled in a questionnaire, comprising sociodemographic characteristics, disease characteristics and questionnaires on physical and mental functioning, coping and satisfaction with assistive devices in possession.

Moreover, data regarding the medical history (disease duration, presence of erosions, current use of diseasemodifying anti-rheumatic drugs (DMARDS) and oral corticosteroids) and a recent $(<3$ months $)$ erythrocyte sedimentation rate (ESR) were abstracted from the medical record by the principal investigator.

\section{Semi-structured interview}

To determine the presence of impairments or activity limitations related to specific assistive devices, the possession and usage and the reasons for usage or non-usage, a semistructured, in-depth interview including a photo presentation of 21 assistive devices that are frequently prescribed in the Netherlands was used. The interview method was used to avoid misunderstandings regarding the possession of specific devices. We hypothesised that by just mentioning the name of a device some patients might not recognise it. In addition, at the time the study was conceived and the protocol developed, no validated self-administered questionnaires or standardised theoretical frameworks for the assessment of individual reasons for usage or non-usage of specific assistive devices were available. As it was thought likely that there would be a variety of experiences, knowledge and opinions of patients playing a role, we decided to use open-ended questions for this specific topic.

According to the ISO9999 classification, the assistive devices were categorised into: orthopaedic footwear (orthopaedic shoes and insoles), personal care (wash and dry bidet, shower chair, sock aid, bathtub seat-elevator), mobility (work chair, rolling walker, mobility scooter, electric wheelchair, bicycle with electric support), house- 
hold (loop scissors, reacher, good-angled grip cutlery, electric can opener) and adaptations for housing (lift chair, stairway chair lift, grab bars, electric bedspring, orthopaedic pillow). Apart from the electric can opener, we did not include consumer products because these may also be commonly used by healthy people. In addition, for the sake of relevance, we did not consider assistive devices costing $€ 20$ or less at the time the study was conducted. With relatively cheap assistive technology, potential savings in case of any improvement in the process of advice, prescription, instruction and delivery as a result of our study would be marginal.

First, with every selected device, patients were asked about the presence of an impairment or activity limitation related to that assistive device in the past 3 months. These problems were scored on a four-point scale ranging from 1 'never' up to 4 'always'.

Patients were then asked whether they had that specific assistive device in possession. For that purpose, a photo presentation of the device was used as an example. For those who did possess that specific assistive device, the following additional information was gathered:

- Frequency of usage in the past 3 months (eight predefined categories: always, several times a day, once a day, several times a week, once a week, once a month, less than once a month, never)

- Individual main reasons for usage (open-ended questions; categories defined afterwards by IGB and TVV: enabling or facilitating specific activity; reduction of pain, fatigue, swelling or compensation for reduced strength; joint protection; recommendation by physician, rheumatologist or health professional)

- Individual main reasons for non-usage (open-ended questions; categories defined afterwards by IGB and TVV: no impairment and/or activity limitation related to device; negative evaluation of device, e.g. did not met expectations, not easy to use, dislike the appearance, lack of fit or comfort; other (having impairment and/or activity limitation but not having reached that stage yet; others peoples' negative opinion; or other impairment or activity limitation not related to device).

Patients could provide more than one activity or reason. Any discrepancies with respect to the categorisation of reasons for usage or non-usage by IGB and TVV were solved by consensus.

Questionnaire and clinical assessment

\section{Sociodemographics}

Sociodemographic variables included age (years), gender, educational level (categorised as low: up to and including lower technical and vocational training; medium: up to and including secondary technical and vocational training; high: up to and including higher technical and vocational training and university) and status of living (living alone or with partner or children).

\section{Disease characteristics}

Disease duration (years) and current use of DMARDS and oral corticosteroids were registered. Comorbidity was assessed using a part of the Dutch Arthritis Impact Measurement Scale II [15], and categorised into two groups: no comorbid conditions and one or more comorbid conditions.

General disease activity was assessed by means of the Modified Disease Activity Score (DAS28 [16]), using the ESR, the 28-joint count and the patients' global assessment of disease activity, measured on a horizontal visual analogue scale (VAS, range 0-100 mm).

The DAS28 was calculated by the following formula: $0.56 \sqrt{ }$ (tender joints $)+0.28 \sqrt{ }$ (swollen joints $)+0.70 \ln ($ ESR $)+$ 0.014 (VAS patients' global assessment of disease activity).

Measures of general pain and fatigue included a horizontal VAS, range $0-100 \mathrm{~mm}$. The left anchors were no pain and no fatigue and the right anchors unbearable pain and severe fatigue, respectively.

\section{Physical and mental functioning}

A validated Dutch version of the RAND 36-item Health Survey (RAND-36 [17]) was used. The RAND-36 contains eight subscales for: physical functioning, social functioning, role limitations (physical problem), role limitations (emotional problem), mental health, vitality, pain and general health perception. Each scale generates a score from 0 to 100, with higher scores indicating better health. The RAND-36 was converted to two summary scales: the physical and mental component summary scales.

\section{Coping}

Coping strategies were assessed by the "coping with rheumatic stressors" questionnaire [18], an arthritis-specific coping questionnaire which measures eight coping strategies directed at the most important chronic stressors of inflammatory rheumatic disease: pain, limitations and dependency. Our study included the measurement of five coping styles related to pain (decreasing activities: eight items), limitations (pacing: ten items, creative solution seeking: eight items) and dependence (accepting: six items, taking others into account: seven items). For each item, patients reported how often they made use of that particular coping strategy $(1=$ seldom or never, $2=$ sometimes, $3=$ 
often, $4=$ very often). The scores were summed up per coping strategy to give a coping strategy score. Higher scores indicate more frequent use of that specific coping strategy.

\section{Satisfaction}

All patients who possessed a device were asked to score their satisfaction with different aspects of that specific device by filling in the D-Quest (Dutch version of the Quebec User Evaluation of Satisfaction with assistive Technology [19]). This questionnaire comprises eight aspects relating to the assistive device (ease of use, effectiveness, comfort, adjustments, weight, durability, dimensions, safety), four aspects relating to the process of providing the assistive devices (service delivery, repairs and servicing, professional services, follow-up services), a general score for satisfaction with the device and a general score for satisfaction with the process of prescription and delivery. Each dimension is scored on five-point scale ranging from 1 'not satisfied at all' to 5 'very satisfied'.

All answers were categorised afterwards in 'satisfied' $(4=$ satisfied and $5=$ very satisfied $)$ 'not satisfied' $(1=$ not satisfied at all, $2=$ not satisfied and $3=$ not satisfied-satisfied).

\section{Statistical analysis}

All analyses were conducted using SPSS 12.0 for Windows (SPSS Chicago IL, USA). Frequency distributions and summary statistics for the full sample as well as for the three hospitals separately were obtained. For categorical data, proportions were calculated; all other data were expressed as median and interquartile ranges (IQR). To analyse differences between the participants of the three hospitals and between participants and non-participants (eligible patients who were excluded, not reached by phone or declined participation) the Mann-Whitney test for continuous variables and the chi square for discrete variables were conducted.

For each of the assistive devices, the usage rates were categorised into three groups: 1 (always, several times a day or once a day); 2 (several times a week or once a week); and 3 (once a month, less then once a month or never). In addition, the median usage rate was computed. Moreover, within each ISO9999 category, the median frequency of usage of all devices in possession in that category was computed.

To determine which variables were associated with usage, firstly univariate logistic regression analysis was performed, with the results expressed as odds ratios with the $95 \%$ confidence interval. The dependent variable was the frequency of usage of assistive devices in a specific category, dichotomised into $>$ median frequency (=frequent use) versus $\leq$ median frequency (=non-frequent use). Only variables that were statistically significantly associated with usage in the univariate analyses ( $p$ value $\leq 0.05)$ were then entered into multivariate logistic regression models with the same dependent variable. To exclude possible confounding, the variables centre, age and gender were entered in the first block, regardless of the statistical significance of the univariate analyses (method forced entry), and in addition the independent variables that reached statistical significance in the univariate analysis were entered in the second block (method stepwise forward).

\section{Results}

Of the 444 patients who were eligible according to the diagnosis of RA in the database and the medical record, $362(82 \%)$ could be reached by phone, whereas 82 patients $(18 \%)$ could not be contacted by phone after a maximum of three attempts. Out of 362 patients, 251 (69\%) agreed to participate in the study. Eleven patients were excluded from the analysis at a later stage due to the absence of all questionnaires or the interview, yielding a total number of 240 participants (54\%) and 204 non-participants $(46 \%)$.

The proportions of participants were similar in the three centres (Leiden 138/242, 57\%; Delft 63/124, 51\%; and The Hague $39 / 78,50 \%, p=0.386$ ). Concerning the characteristics of participants and non-participants, the proportion of female patients was significantly higher among participants than among non-participants $(185 / 240,77 \%$ and $125 / 204$, $61 \%$, respectively; $p<0.001)$. This difference was seen in all three centres (data not shown).

\section{Patient characteristics}

The sociodemographic and disease characteristics and coping strategies of the 240 participants are presented in Table 1.

Possession and usage of assistive devices

Of the 240 subjects enrolled in the study, $213(89 \%)$ had at least one assistive device in possession (median number of devices 3.0, IQR 3.0). Table 2 shows the frequencies of possession for all 21 devices and for the categories of devices. The assistive devices that were most frequently in possession were: grab bars (44\%), orthopaedic shoes $(38 \%)$, orthopaedic pillow $(32 \%)$, shower chair $(29 \%)$ and electric can opener $(27 \%)$. There were no significant differences between the three hospitals regarding the frequencies of possession (data not shown). 
Table 1 Characteristics of 240 patients with rheumatoid arthritis participating in a study on the possession and usage of assistive devices. Results presented as median (IQR), unless specified otherwise

Total $(N=240)$

Sociodemographics

Age (years)

$63(18)$

Female, $n(\%)$

$185(77)$

Living alone, $n(\%)$

$53(22)$

Level of education, $n(\%)$

Low

$102(43)$

Medium

$107(45)$

High

$25(10)$

Disease characteristics

Disease duration (years)

Medication, $n(\%)$

Oral corticosteroids

$9(12.5)$

$42(18)$

DMARDs

$215(90)$

$124(52)$

Comorbidity, $n(\%)$

$4.1(1.7)$

$33(36)$

$3.3(3.3)$

$5.0(5)$

Fatigue (VAS; $0-10 \mathrm{~cm}$ )

$48.7(41.9)$

Physical and mental functioning

RAND-36 physical component (0-100)

RAND-36 mental component (0-100)

$74.6(28.7)$

Coping strategies (CORS)

Decreasing activity $(8-31)$

$19(6)$

Pacing (10-40)

$28(8)$

Creative solution seeking (8-32) 22 (6)

Accepting (6-24)

$14(6)$

Taking others into account (7-28)

The main reason for not using orthopaedic footwear was a negative evaluation (ease of use, dislike the appearance, did not fit well, comfort). The main reasons for not using an assistive device in the other categories were 'no impairment or activity limitation related to the device'. All these reasons for usage and non-usage mentioned in the interviews were covered by the previously mentioned potential variables associated with usage and were therefore not entered separately into the regression analyses.

Satisfaction

In Table 4, the proportions of patients being 'satisfied-very satisfied' are shown for those devices that were in possession by 50 patients or more. Overall, satisfaction with the devices and with the providing process were high, with, according to the general scores, $75 \%$ or more of the patients being satisfied or very satisfied with the different aspects of the device and the providing process. Regarding specific aspects of satisfaction, satisfaction rates were the lowest for orthopaedic shoes, specifically with the aspects dimensions $(50 ; 67 \%)$, weight $(51 ; 68 \%)$ and comfort $(54$; $71 \%$ ). Patients were also less satisfied with the aspect adjustments of the electric can opener $(24 ; 60 \%)$, implying that it was not suitable for different kinds of cans.

Factors associated with the usage of assistive devices

$D M A R D$ Disease-modifying anti-rheumatic drugs, VAS visual analogue scale, CORS coping with rheumatic stressors

With respect to usage, the median frequency of usage varied between 'always' $(=1.0)$ for orthopaedic shoes and 'less than once a month' (=7.0) for bathtub seat-elevator.

For devices in possession by 50 patients or more, the proportions of patients who indicated that they never used the device were: $11 / 132$ (12\%) for orthopaedic shoes, 4/53 (8\%) for orthopaedic insoles, 13/69 (19\%) for a shower chair, 13/65 (20\%) for an electric can opener, 24/105 (23\%) for grab bars, $11 / 56(20 \%)$ and $12 / 76(16 \%)$ for an orthopaedic pillow.

Reported reasons for usage and non-usage

In Table 3, the main reasons for using or not using an assistive device reported by the patients are presented. In all five categories of assistive devices, the main reasons for usage were 'enabling or facilitating specific activities' or 'reduction of pain, fatigue, swelling or compensation for reduced strength', both among frequent users and nonfrequent users.

In addition, adaptations for housing and devices for household and personal care were also used for safety reasons.

The findings of the multivariate logistic analyses for usage of assistive devices are presented in Table 5. The data indicate that pain and the presence of an impairment or limitation related to a specific device were positively associated with frequent usage within the categories orthopaedic footwear, personal care and adaptations for housing.

Self-efficacy was positively associated with usage in the categories mobility and household, general satisfaction with the usage in the categories orthopaedic footwear and personal care and satisfaction with the prescription and provision process with usage in the category adaptations for housing. In addition, positive outcome expectations were negatively associated with usage within the category adaptations for housing.

The total variance explained by the factors entered in the models was $12 \%$ for mobility, $24 \%$ for household, $34 \%$ for adaptations for housing, $38 \%$ for personal care and $47 \%$ for orthopaedic footwear.

\section{Discussion}

This cross-sectional study demonstrates that nearly $90 \%$ of the patients with RA possessed one or more of a selection 
Table 2 Frequency of possession and usage of assistive devices in patients with rheumatoid arthritis $(N=240)$

\begin{tabular}{|c|c|c|c|c|c|c|c|c|c|}
\hline & & & Possessio & & & Usage & & & \\
\hline ISO & 9999 category & & $n(\%)$ & $n(\%)^{\mathrm{a}}$ & Median & Median & Daily, & Weekly, & Monthly or \\
\hline 06 & Orthopaedic & Orthopaedic shoes & $91(38)$ & $132(55)$ & $6.0(7.5)$ & $1.0(1.0)$ & $72(80)$ & $6(7)$ & $12(13)$ \\
\hline & footwear & Orthopaedic insoles & $53(22)$ & & $5.0(7.0)$ & $1.0(2.0)$ & $43(81)$ & $4(8)$ & $6(11)$ \\
\hline 09 & Personal care & Wash and dry bidet & $13(5)$ & & $3.5(3.6)$ & $2.0(1.0)$ & $11(85)$ & $1(8)$ & $1(8)$ \\
\hline & & Shower chair & $69(29)$ & $82(34)$ & $4.0(7.5)^{\mathrm{d}}$ & $4.0(3.0)$ & $31(46)$ & $19(28)$ & $18(27)$ \\
\hline & & Sock aid & $3(1)$ & & $9.5(1.0)^{\mathrm{d}}$ & $7.0(7.0)^{\mathrm{d}}$ & $1(33)$ & 0 & $2(67)$ \\
\hline & & Bathtub seat-elevator & $11(5)$ & & $5.0(8.0)$ & $7.0(4.0)$ & $2(18)$ & $3(27)$ & $6(55)$ \\
\hline 12 & Mobility & Work chair & $27(11)$ & & $10.0(8.0)$ & $2.0(1.0)$ & $21(78)$ & $3(11)$ & $3(11)$ \\
\hline & & Rolling walker & $37(15)$ & & $2.0(3.0)$ & $4.0(6.0)$ & $17(47)$ & $8(22)$ & $11(31)$ \\
\hline & & Mobility scooter & $21(9)$ & $85(35)$ & $4.5(6.6)$ & $4.0(1.0)$ & $9(47)$ & $6(32)$ & $4(21)$ \\
\hline & & Electric wheelchair & $1(0)$ & & $10.0(0)^{\mathrm{c}}$ & $2.0(0)^{\mathrm{c}}$ & $1(100)$ & 0 & 0 \\
\hline & & Wheelchair & $29(12)$ & & $4.5(7.9)$ & $6.5(3.0)$ & $1(4)$ & $6(32)$ & $21(75)$ \\
\hline & & Bicycle with electric support & $15(6)$ & & $3.0(3.0)$ & $4.0(4.0)$ & $7(47)$ & $3(20)$ & $5(33)$ \\
\hline 15 & Household & Loop scissors & $17(7)$ & & $10.0(6.5)$ & $5.0(3.0)$ & $3(18)$ & $9(53)$ & $5(29)$ \\
\hline & & Reacher & $27(11)$ & $104(43)$ & $5.0(6.5)$ & $3.0(4.0)$ & $23(64)$ & $4(11)$ & $9(25)$ \\
\hline & & Good-angled grip cutlery & $36(15)$ & & $6.0(7.5)$ & $5.0(3.0)$ & $6(22)$ & $11(41)$ & $10(37)$ \\
\hline & & Electric can opener & $65(27)$ & & $10.0(14.3)$ & $6.0(2.0)$ & $4(6)$ & $24(37)$ & $37(57)$ \\
\hline 18 & Adaptations for & Lift chair & $36(15)$ & & $3.5(5.5)$ & $2.0(0.0)$ & $31(91)$ & 0 & $3(9)$ \\
\hline & housing & Stairway chair lift & $15(6)$ & & $4.0(8.8)$ & $2.0(0.0)$ & $12(86)$ & 0 & $2(14)$ \\
\hline & & Grab bars & $105(44)$ & $170(71)$ & $5.0(8.0)$ & $3.0(6.0)$ & $66(64)$ & $8(8)$ & $30(29)$ \\
\hline & & Electric bedspring & $56(23)$ & & $4.5(8.0)$ & $4.0(4.0)$ & $25(46)$ & $10(18)$ & $20(36)$ \\
\hline & & Orthopaedic pillow & $76(32)$ & & $5.0(7.0)$ & $1.0(2.0)$ & $60(79)$ & $4(5)$ & $12(16)$ \\
\hline
\end{tabular}

${ }^{\mathrm{a}}$ Number (\%) possessing one or more assistive devices within category

${ }^{\mathrm{b}}$ Duration of possession in years

${ }^{\mathrm{c}}$ Frequencies usage: 1 'always'; 2 'several times a day'; 3 'once a day'; 4 'several times a week'; 5 'once a week'; 6 'once a month'; 7 'less than once a month'; 8 'never'; Daily = always + several times a day + once a day; Weekly = several times a week + once a week; Monthly or never $=$ once a month + less than once a month + never

${ }^{\mathrm{d}}$ Range instead of IQR

of 21 assistive devices. Usage rates varied among the various categories of assistive devices, with on average usage rates being daily in the categories orthopaedic footwear and adaptations for housing and weekly in the categories personal care, mobility or household. The proportions of patients who had completely abandoned an assistive device ranged between $8 \%$ and $23 \%$.

The total possession rates for assistive devices in our study were however slightly higher than the maximum rates reported in previous studies. In previous studies, proportions of patients possessing one or more assistive devices varied between $34 \%$ and $78 \%$ [3, 5-7]. This difference could probably be explained by variation in the number of assistive devices or groups of assistive devices that were taken into account [3, 5-7] or by differences among patient populations (age and disease duration [3, 6]). Moreover, it has been demonstrated before that possession of assistive devices may vary along with country-related health care systems [5].

Similar to the results of the present study, house adaptations and mobility devices were found to be the most prevalent assistive devices in two previous studies $[5,6]$.
Usage rates as reported in the present study are hard to compare with those of other studies concerning assistive devices in general $[2,3,7,12]$, mainly because different definitions of usage have been used. None of the previous studies assessed the absolute frequency of usage as employed in the present study. Apart from different methods to measure usage, different assistive devices or groups of assistive devices, patient populations and duration of follow-up have been used.

The present study is unique in the fact that the main reasons for using or not using devices were assessed. The most important reason to use a device was to enable or facilitate an activity which otherwise would be impossible or difficult, whereas not having problems with the activity related to the device appeared to be the main reason for not using the devices. This result underlines the importance of assistive devices as a compensation for activity limitations. Our findings confirm the results of previous research, where functional limitations and disease severity were found to predict usage $[2,7,12]$. The relationship between specific limitations or problems and the usage of adaptive 


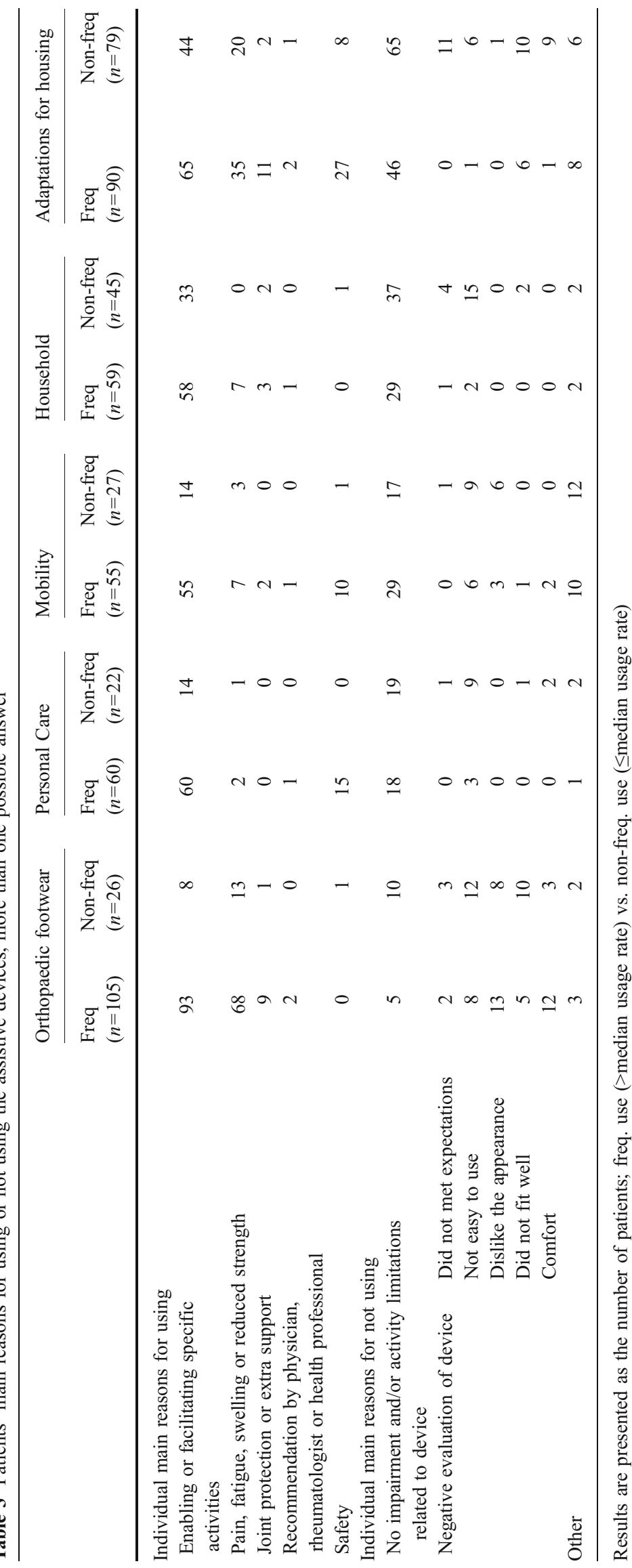


Table 4 Patients' satisfaction with the assistive device and providing process for devices in possession by more than 50 patients

\begin{tabular}{|c|c|c|c|c|c|c|c|}
\hline & $\begin{array}{l}\text { Orthopaedic } \\
\text { shoes }\end{array}$ & $\begin{array}{l}\text { Orthopaedic } \\
\text { insoles }\end{array}$ & Shower chair & $\begin{array}{l}\text { Electr. can } \\
\text { opener }\end{array}$ & Grab bars & Electr. bedspring & $\begin{array}{l}\text { Orthopaedic } \\
\text { pillow }\end{array}$ \\
\hline \multicolumn{8}{|l|}{ Satisfaction device } \\
\hline Dimensions & $50 / 75(67)$ & $40 / 44(91)$ & $53 / 58(91)$ & $50 / 57(88)$ & $82 / 87(94)$ & $42 / 43(98)$ & $53 / 65(82)$ \\
\hline Weight & $51 / 75(68)$ & $42 / 43(98)$ & $48 / 50(96)$ & $48 / 57(84)$ & $52 / 54(96)$ & $26 / 30(87)$ & $57 / 64(89)$ \\
\hline Adjustments & $44 / 53(83)$ & $18 / 22(82)$ & $46 / 49(94)$ & $24 / 40(60)$ & $44 / 49(90)$ & $39 / 42(93)$ & $20 / 26(77)$ \\
\hline Safety & $58 / 66(82)$ & 28/30 (93) & $49 / 55(89)$ & $49 / 56(88)$ & $81 / 84(96)$ & 41/41 (100) & $39 / 40(98)$ \\
\hline Durability & $65 / 76(86)$ & $33 / 43(77)$ & $54 / 57(95)$ & $50 / 55(91)$ & $82 / 84(98)$ & $40 / 42(95)$ & $56 / 62(90)$ \\
\hline Ease of use & $58 / 74(78)$ & $39 / 43(91)$ & $51 / 58(95)$ & $46 / 57(81)$ & $81 / 86(94)$ & $42 / 43(98)$ & $57 / 65(88)$ \\
\hline Comfort & $54 / 76(71)$ & $39 / 43(91)$ & $48 / 58(83)$ & $49 / 57(86)$ & $79 / 85(93)$ & $42 / 43(98)$ & $53 / 66(80)$ \\
\hline Effectiveness & $61 / 75(81)$ & $38 / 44(86)$ & $50 / 57(88)$ & $48 / 56(86)$ & $82 / 87(94)$ & 43/43 (100) & $56 / 65(86)$ \\
\hline General score assistive device & $58 / 77(75)$ & $38 / 44(86)$ & $50 / 59(85)$ & $48 / 57(84)$ & $84 / 89(94)$ & $41 / 44(93)$ & $56 / 66(85)$ \\
\hline \multicolumn{8}{|l|}{ Satisfaction providing process } \\
\hline Service delivery & $61 / 75(81)$ & $39 / 43(91)$ & $45 / 52(87)$ & $16 / 19(84)$ & $59 / 65(91)$ & $17 / 21(81)$ & $25 / 31(81)$ \\
\hline Repairs and servicing & $52 / 61(85)$ & $24 / 30(80)$ & $29 / 32(91)$ & $16 / 17(94)$ & $31 / 34(91)$ & $15 / 18(83)$ & $16 / 18(89)$ \\
\hline Professional services & $60 / 75(80)$ & $37 / 43(86)$ & $44 / 49(90)$ & $15 / 18(83)$ & $50 / 61(82)$ & $18 / 20(90)$ & $27 / 30(90)$ \\
\hline Follow-up services & $63 / 70(90)$ & $28 / 35(80)$ & $37 / 41(90)$ & $10 / 11(91)$ & $48 / 54(89)$ & $17 / 19(90)$ & $16 / 19(84)$ \\
\hline General score providing process & $62 / 74(84)$ & $37 / 43(86)$ & $46 / 50(92)$ & $14 / 17(82)$ & $57 / 65(88)$ & $21 / 23(91)$ & $29 / 34(85)$ \\
\hline
\end{tabular}

Results are presented as number (satisfied-very satisfied) / number responders (\%)

devices could imply that the prescription of assistive devices should preferably be confined to those patients that currently have or are likely in the future to have an impairment or activity limitation related to the device. The previously observed relationships between usage and the sociodemographic variables age and gender are contradic- tory $[2,7,11]$. In this study, neither an association of usage with age nor with gender could be confirmed.

With respect to satisfaction with the device and the providing process, it was found that the majority of the patients were satisfied or very satisfied with the devices as well as with the providing process, so there appears to be

Table 5 Multivariate analyses: factors significantly associated with the usage of assistive devices for ISO9999 categories separately

\begin{tabular}{|c|c|c|c|c|c|}
\hline & $\begin{array}{l}\text { Orthopaedic footwear } \\
\text { OR }(95 \% \mathrm{CI})^{\mathrm{a}}\end{array}$ & $\begin{array}{l}\text { Personal Care } \\
\text { OR }(95 \% \mathrm{CI})^{\mathrm{a}}\end{array}$ & $\begin{array}{l}\text { Mobility } \\
\text { OR }(95 \% \mathrm{CI})^{\mathrm{a}}\end{array}$ & $\begin{array}{l}\text { Household } \\
\text { OR }(95 \% \mathrm{CI})^{\mathrm{a}}\end{array}$ & $\begin{array}{l}\text { Adaptations for } \\
\text { housing OR }(95 \% \mathrm{CI})^{\mathrm{a}}\end{array}$ \\
\hline Pain (VAS; 0-100 mm) & & $1.7(1.2-2.5)$ & & & \\
\hline $\begin{array}{l}\text { Impairment or activity limitation related } \\
\text { to a specific assistive device }\end{array}$ & $2.6(1.2-5.5)$ & & & & $2.8(1.3-5.9)$ \\
\hline Coping: accepting & & & & & $1.1(1.02-1.3)$ \\
\hline Outcome expectations & & & & & $0.4(0.2-0.8)$ \\
\hline Self-efficacy & & & $2.5(1.2-5.1)$ & $2.4(1.2-4.7)$ & \\
\hline Satisfaction assistive device (D-Quest) & $20.3(4.6-90.1)$ & $5.0(1.0-26.2)$ & & & \\
\hline Satisfaction providing process (D-Quest) & & & & & $9.1(2.4-34.5)$ \\
\hline Duration of possession (years) & & & & $0.9(0.9-0.99)$ & \\
\hline $\begin{array}{l}\text { Number of devices in possession } \\
\text { in the category }\end{array}$ & $0.1(0.02-0.64)$ & & & $2.7(1.2-6.2)$ & \\
\hline$R^{2}$ & 0.47 & 0.38 & 0.12 & 0.24 & 0.34 \\
\hline
\end{tabular}

Independent variables that were significantly associated with usage in the univariate analyses $(p \leq 0.05)$ were entered into a multivariate logistic regression model: ISO6: DAS28, impairment or activity limitation related to device, RAND-36 physical, coping: taking others into account, satisfaction assistive device, satisfaction providing process, number of devices in possession. ISO9: level of education, pain (VAS), fatigue (VAS), disease activity (VAS), impairment or activity limitation related to device, RAND-36 physical and mental, satisfaction assistive device. ISO12: self-efficacy. ISO15: impairment or activity limitation related to device, self-efficacy, duration of possession, number of devices in possession. ISO18: pain (VAS), fatigue (VAS), impairment or activity limitation related to device, RAND-36 physical and mental, coping: decreasing activity, creative solution seeking and accepting, outcome expectations, satisfaction assistive device, satisfaction providing process. Freq. use (>median usage rate) vs. non-freq. use ( $\leq$ median usage rate)

$V A S$ Visual analogue scale, D-Quest Dutch version of the Quebec User Evaluation of Satisfaction with assistive Technology, $R^{2}$ Nagelkerke $R^{2}=$ pseudo measure for proportion explained variance

${ }^{a}$ Adjusted odds ratios with $95 \%$ confidence intervals, logistic regression with centre, age and gender entered first and significant independent variables entered second 
little room for improvement. The somewhat lower satisfaction rates for orthopaedic footwear could mean that a thorough evaluation of the design of orthopaedic shoes is desirable. According to the comments made by the patients, the weight, comfort and the fitting of the orthopaedic shoes could be improved. The results of our study stress the need for the further development of orthopaedic footwear design, including functional aspects, comfort and aesthetics.

The results of this study show that positive outcome expectations appeared to be associated with less-frequent usage in the category adaptations for housing. A significant association between outcome expectations and usage was absent in all other categories. Regarding the interpretation of the relationship between outcome expectations and usage, it should be noted that this study had a crosssectional design, and the recall of outcome expectations may have been biased by positive or negative experiences with the device. To explore the relation between the outcome expectations and usage, a prospective design would have been more preferable.

In our study, reasons for usage and non-usage were listed by means of in-depth interviews, with the patients' responses being categorised afterwards. This approach was chosen in the absence of a list of topics or questionnaires specifically addressing assistive device utilisation. After this study had been conducted, there have been advances in the field of assistive technology outcomes research, such as the development of a model for assistive technology usage [20]. According to this model, the intention to use assistive devices is a function of perceived advantages in terms of effectiveness, efficiency, satisfaction and subjective well-being and might be modified by personal characteristics, task, strength of the assistive device intervention (including characteristics of the device and associated services) and environmental factors [20,21]. Until now, this model has not been validated. It appears, however, that the factors that were found to be significantly associated with the usage of assistive devices in the present study would fit well into this model.

The present study has a number of other limitations. First, despite the fact that the response rate was satisfactory and three centres were included, it cannot be excluded that patients who participated were different from the general population of RA patients. For the comparisons between participants and non-participants, it was indeed found that the proportion of female patients was higher among the participants. It could be hypothesised that patients with assistive devices in possession could have been more willing to participate or that patients who are old or have serious disabilities or working patients might be less likely to participate in a trial where the completion of extensive questionnaire and an outpatient visit is required. Moreover, with the interview, we used a photo presentation of only one type of the specific assistive device. It can therefore not be totally ruled out that patients did not recognise it in case they possessed another type. In addition, patients were not explicitly asked to take their devices with them to the interview, so that neither the possession nor the adequate usage of the device could be systematically verified. The cross-sectional design of this study also precludes definite conclusions on the exact moment of abandonment of the assistive devices. For those specific research questions, a prospective design is preferable. Moreover, although all analyses were adjusted for each centre, it cannot be totally ruled out that there have been general differences in the process of advice, prescription, delivery and evaluation among the three centres or even among rheumatologists or other health professionals within one centre. In this study, no detailed information on the prescription process, including, e.g. group education sessions or training procedures, was gathered. The reason for this lies in the fact that most patients would have more than one assistive device and would have to give details on the prescription process by recall, while the average duration of possession was considerable. For a profound analysis of the impact of the prescription process on the usage of assistive devices, an additional, prospective study taking into account all the details of the prescription process would be needed.

In conclusion, nearly $90 \%$ of the patients with RA possessed one or more of a selection of 21 assistive devices. Less than $23 \%$ of these devices in possession were abandoned and satisfaction with the devices and providing process was high. Factors associated with usage varied among categories and comprised, apart from the number of devices, variables related to health status, aspects of satisfaction with the device or related services or self-efficacy. The latter finding underlines the need for a structural evaluation of the outcomes of assistive devices by the prescribing health professional or supplier in every individual case.

Acknowledgements This study was financially supported by the Leiden University Medical Center (grant nr. p01.188). We want to thank all participating patients. Furthermore, we want to thank L. Duymaer, K. Collee, J. van Aken, M. Vegter and A. Chorus for their collaboration in this study with respect to the selection of the assistive devices.

Disclosures None.

Open Access This article is distributed under the terms of the Creative Commons Attribution Noncommercial License which permits any noncommercial use, distribution, and reproduction in any medium, provided the original author(s) and source are credited.

\section{References}

1. Mann WC, Hurren D, Tomita M (1995) Assistive devices used by home-based elderly persons with arthritis. Am J Occup Ther 49 (8):810-820 
2. Rogers JC, Holm MB (1992) Assistive technology device use in patients with rheumatic disease: a literature review. Am J Occup Ther 46(2): 120-127

3. Thyberg I, Hass UA, Nordenskiold U, Skogh T (2004) Survey of the use and effect of assistive devices in patients with early rheumatoid arthritis: a two-year follow up of women and men. Arthritis Rheum 51(3):413-421

4. Veehof M, Taal E, Rasker J, Lohmann J, Van de LM (2006) Possession of assistive devices is related to improved psychological well-being in patients with rheumatic conditions. J Rheumatol 33(8):1679-1683

5. Veehof MM, Taal E, Rasker JJ, Lohmann J, van de Laar MA (2006) What determines the possession of assistive devices among patients with rheumatic diseases? The influence of the countryrelated health care system. Disabil Rehabil 28(4):205-211

6. van der Heide A, Jacobs JW, Albada-Kuipers GA, Kraaimaat FW, Geenen R, Bijlsma JW (1993) Self report functional disability scores and the use of devices: two distinct aspects of physical function in rheumatoid arthritis. Ann Rheum Dis 52(7):497-502

7. Van der Esch M, Heijmans M, Dekker J (2003) Factors contributing to possession and use of walking aids among persons with rheumatoid arthritis and osteoarthritis. Arthritis Rheum 49 (6):838-842

8. Nordenskiold U, Grimby G, Dahlin-Ivanoff S (1998) Questionnaire to evaluate the effects of assistive devices and altered working methods in women with rheumatoid arthritis. Clin Rheumatol 17 (1):6-16

9. Nordenskiold U (1994) Evaluation of assistive devices after a course in joint protection. Int $\mathrm{J}$ Technol Assess Health Care 10 (2):293-304

10. Nordenskiold U, Grimby G (1997) Assessments of disability in women with rheumatoid arthritis in relation to grip force and pain. Disabil Rehabil 19(1):13-19

11. Haworth RJ (1983) Use of aids during the first three months after total hip replacement. Br J Rheumatol 22(1):29-35
12. Hollings EM, Haworth RJ (1978) Supply and use of aids and appliances: a study of 119 patients with rheumatoid arthritis. Br J Occup Ther 41:336-339

13. Arnett FC, Edworthy SM, Bloch DA, McShane DJ, Fries JF, Cooper NS et al (1988) The American Rheumatism Association 1987 revised criteria for the classification of rheumatoid arthritis. Arthritis Rheum 31(3):315-324

14. Smolen JS, Breedveld FC, Eberl G, Jones I, Leeming M, Wylie GL et al (1995) Validity and reliability of the twenty-eight-joint count for the assessment of rheumatoid arthritis activity. Arthritis Rheum 38(1):38-43

15. Riemsma RP, Taal E, Rasker JJ, Houtman PM, Van Paassen HC, Wiegman O (1996) Evaluation of a Dutch version of the AIMS2 for patients with rheumatoid arthritis. $\mathrm{Br} \mathrm{J}$ Rheumatol 35(8): $755-760$

16. Prevoo ML, t Hof MA, Kuper HH, van Leeuwen MA, van de Putte LB, van Riel PL (1995) Modified disease activity scores that include twenty-eight-joint counts. Development and validation in a prospective longitudinal study of patients with rheumatoid arthritis. Arthritis Rheum 38(1):44-48

17. Hays RD, Sherbourne CD, Mazel RM (1993) The RAND 36-Item Health Survey 1.0. Health Econ 2(3):217-227

18. van Lankveld WGJM, Näring GWB, van der Staak CPF, van de Putte LB (1993) Coping with chronic stressors of rheumatoid arthritis. Katholieke Universiteit Nijmegen, Nijmegen

19. Demers L, Wessels R, Weiss-Lambrou R, Ska B, De Witte LP (2001) Key dimensions of client satisfaction with assistive technology: a cross-validation of a Canadian measure in The Netherlands. J Rehabil Med 33(4):187-191

20. Lenker JA, Paquet VL (2004) A new conceptual model for assistive technology outcomes research and practice. Assist Technol 16:1-10

21. Veehof MM, Taal E, Willems MJ, van de Laar MAFJ (2008) Determinants of the use of wrist working splints in rheumatoid arthritis. Arthritis Rheum 59:531-536 